\title{
Grosse Nachfrage zu ambulanten Tarifauskünften
}

\section{Patrick Müller}

Leiter Abteilung Ambulante Versorgung und Tarife

\section{Die Abteilung Ambulante Versorgung und Tarife beantwortet den Mitgliedern der}

FMH Fragen zu ambulanten Arzttarifen. Antworten auf häufig gestellte Fragen

(FAQ) sind auch auf der Website zu finden. Insbesondere die Inkraftsetzung des zweiten Tarifeingriffes per 1. Januar 2018 im Bereich KVG löste bei Ärztinnen und Ärzten eine Flut von Fragen aus.

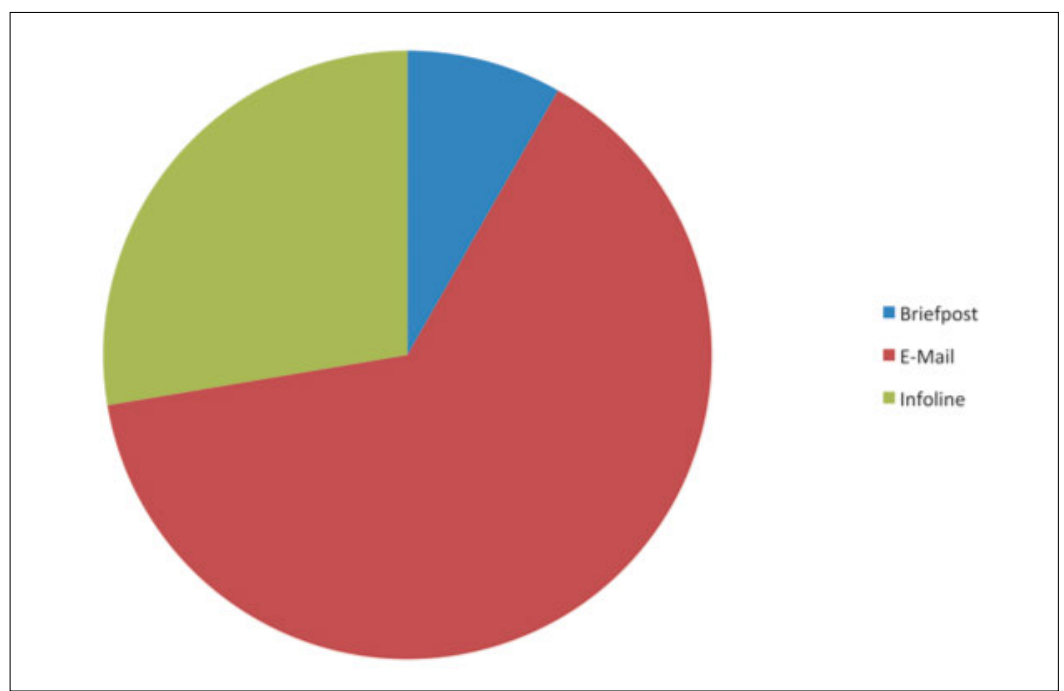

Abbildung 1: Anfrageart der eingehenden Tarifanfragen: Rund zwei Drittel treffen per E-Mail ein.

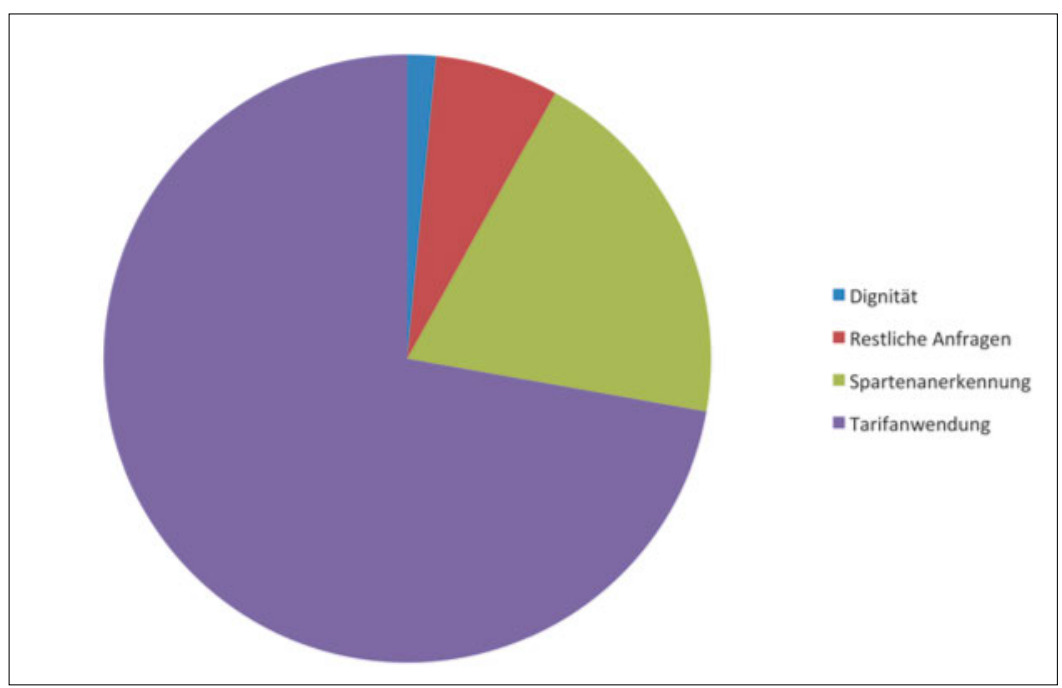

Abbildung 2: Rund 70 Prozent der Anfragen zum TARMED betreffen Fragen zur Tarifanwendung.
Die Mitglieder der FMH können ihre Fragen zu den ambulanten Tarifen entweder per Briefpost, E-Mail oder Infoline der Abteilung Ambulante Versorgung und Tarife stellen. Die Tarifexperten beantworten die Anfragen aufgrund ihrer Ausbildung und Erfahrungen nach bestem Wissen. Gleichzeitig bietet die Website der FMH unter der Rubrik «Ambulante Tarife» weitere Möglichkeiten, sich über die ambulanten Tarife zu informieren. Neben Antworten auf häufig gestellte Fragen (FAQ) sind dort tarifarische Neuigkeiten, Hintergrundinformationen oder auch der TARMEDBrowser zum Herunterladen aufgeschaltet. Die Tarifexperten geben Auskünfte zur Anwendung des Tarifwerkes TARMED, Analysenliste, Spezialitätenliste und der Mittel- und Gegenstände-Liste (MiGeL).

\section{Einblick in die Statistik der Tarif- auskünfte}

Die typische Tarifanfrage wird von einem Arzt per E-Mail in deutscher Sprache zur Anwendung des TARMED gestellt. Pro Monat stellen die FMH-Mitglieder zwischen 180 und 220 Anfragen. Davon gelangen rund zwei Drittel per E-Mail an die Tarifexperten, und rund 90 Prozent davon betreffen das Tarifwerk TARMED. 70 Prozent aller Fragen zum TARMED betreffen die Tarifanwendung, und die restlichen 30 Prozent verteilen sich auf Anfragen zu Dignitäten, Spartenanerkennungen (z.B. Praxis-OP, delegierte Psychotherapie) und andere.

Die durchschnittliche Bearbeitungsdauer einer Anfrage beträgt 15 Minuten. Rund zwei Drittel aller Anfragen werden von einer Ärztin oder einem Arzt gestellt, ein Drittel von einer Medizinischen Praxisassistentin (MPA) oder einer Medizinischen Praxiskoordinatorin (MPK). 


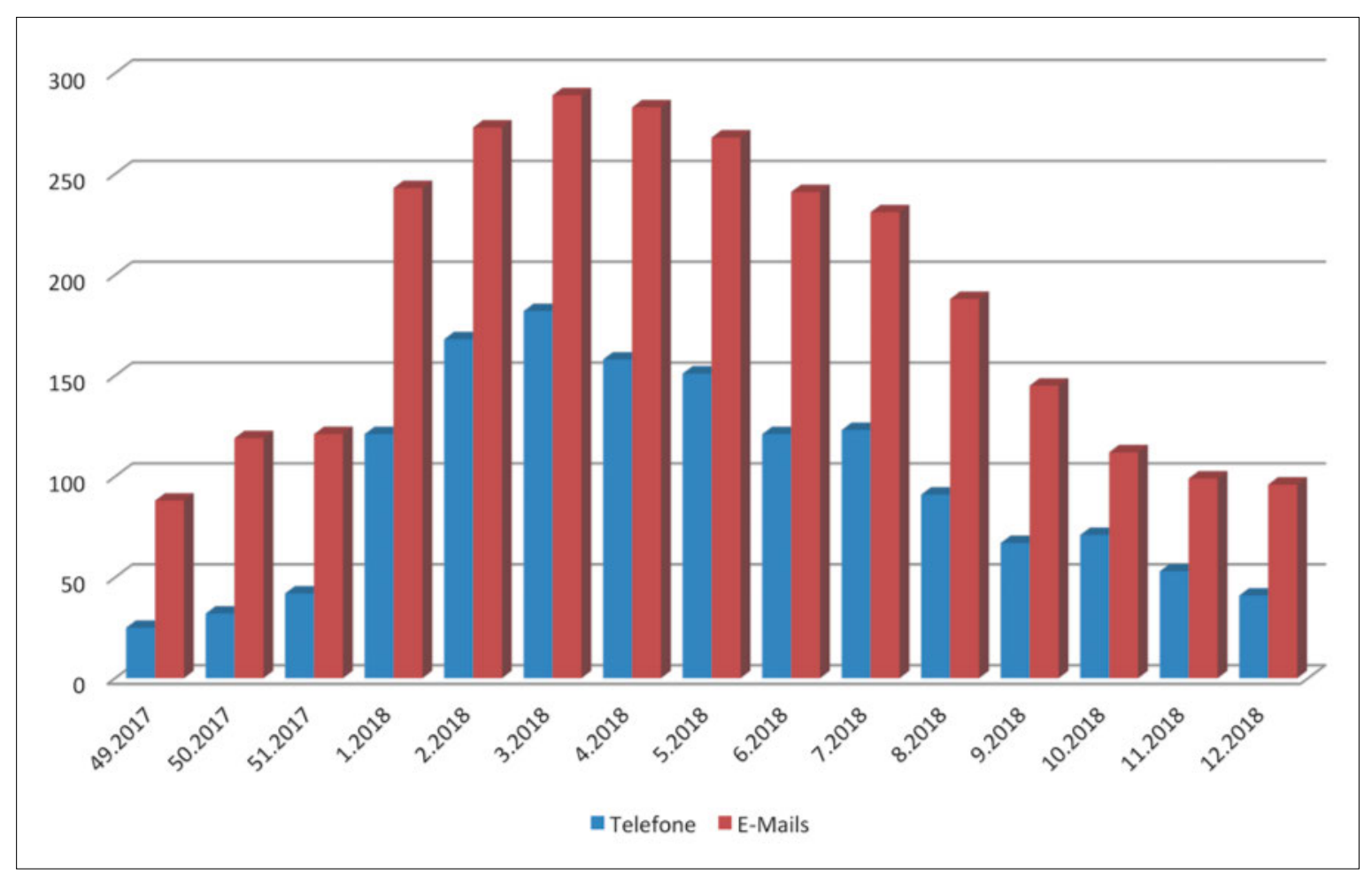

Abbildung 3: Anfragen im Zusammenhang mit dem zweiten Tarifeingriff des Bundesrates in den TARMED. Darstellung entspricht dem Zeitraum von Anfang Dezember 2017 (KW 49) bis Ende März 2018 (KW 12).

\section{Der zweite Tarifeingriff löste einen Ansturm auf die Infoline aus}

Der Bedarf nach aufklärenden und unterstützenden Antworten zur Anwendung des verordneten TARMEDTarifs per 1. Januar 2018 war in den ersten Monaten des Jahres sehr gross. Bereits ab Mitte Dezember 2017 konnte eine signifikante Zunahme der schriftlichen Anfragen (E-Mail) und Telefonate an die Infoline beobachtet werden. Deshalb wurde die Infoline im Monat Januar 2018 auf drei wöchentliche Zeitfenster und in den Monaten Februar und März auf zwei Zeitfenster pro Woche aufgestockt. Alleine im Januar 2018 konnten die Mitarbeitenden der Abteilung Ambulante Versorgung und Tarife rund 650 Telefonate führen und 1100 E-Mails beantworten. Dies entspricht rund 30 Telefongesprächen und 52 E-Mails pro Arbeitstag.

Die vielen Anfragen zeigen, dass die vom Bundesamt für Gesundheit BAG durchgeführten Anpassungen des
TARMED viele Fragen und Unklarheiten hervorbringen. Die Anwendung der verordneten Tarifstruktur ist damit eindeutig komplexer, unklarer und unübersichtlicher geworden. Die Differenzierung der zahlreichen Tarifpositionen führt u.a. auch dazu, dass die Rechnungen unnötig länger werden und die vom Bundesrat geforderte Transparenz der TARMED-Rechnungen damit nicht erfüllt werden kann.

\section{Haben Sie Fragen zu TARMED oder anderen ambulanten Arzttarifen?}

Als Mitglied der FMH erreichen Sie uns per E-Mail unter tarife. ambulant[at]fmh.ch oder telefonisch auf unserer Infoline:

$0900340340 *$

Montags 9 bis $12 \mathrm{Uhr}$

* Im Festnetz: CHF 0.08/Min. 